\section{Responsabilidad sanitaria en odontólogos. Casuística de casos evaluados en el Servicio Médico Legal de Chile}

\author{
KARLA MOSCOSO MATUS ${ }^{1, a}$, PÍA SMOK VÁSQUEZ ${ }^{2, b}$
}

\section{Professional liability claims against dentists}

Background: The frequency and features of malpractice lawsuits against dentists in Chile are not well known. Aim: To determine the magnitude and frequency of professional liability claims against dentists. Material and Methods: A retrospective analysis of the Medical Liability Unit of the Legal Medical Service of Chile database. This public organization deals with most professional liability claims in Chile. Results: Between 2007 and 2012, 3,990 expert opinions about liability of health care professionals were requested. Odontology was the fifth specialty most commonly sued and dentists, the second most frequently sued professionals. Sixty nine percent of cases originated in private clinics, which is coincident with a higher frequency of dentists working in private practice. Most petitioners were adult women and most claims originated from surgical interventions and infections. In 35\% of claims against dentists, a violation of Lex Artis was confirmed, compared with $9 \%$ of all expert opinions that generated in the unit. Conclusions: Claims against dentists are more common than previously thought and these professionals should adopt preventive measures to avoid them.

(Rev Med Chile 2015; 143: 345-351)

Key words: Dentistry; Legislation, medical; Malpractice.

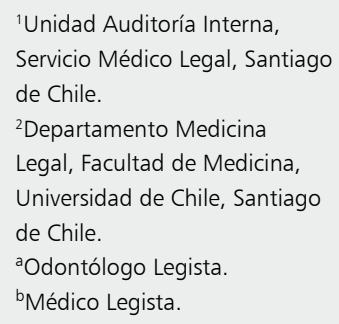

Recibido el 15 de septiembre de 2014, aceptado el 12 de enero de 2015 .

Correspondencia a: Dra. Karla Moscoso Matus Teatinos \#240, Santiago Teléfono: 02-28929200 kmoscoso@gmail.com
$\mathrm{E}$ 1 Servicio Médico Legal de Chile (SML) es una entidad dependiente del Ministerio de Justicia, creada en el año 1915. La Ley Orgánica No 20.065 que lo rige, le otorga, entre otras, la responsabilidad de asesorar técnica y científicamente a los órganos de la justicia a través de la realización de pericias médico legales, así como la de ejercer la tuición técnica de los organismos o personas que ejerzan estas funciones ${ }^{1}$.

Dentro de su estructura orgánica, se encuentra el Departamento de Clínica Forense, en cuya Unidad de Responsabilidad Médica se realizan los peritajes solicitados por el Ministerio Público y Tribunales de Justicia, relativos a Responsabilidad Profesional sanitaria en causas penales y/o civiles, informes que coloquialmente son conocidos como "Negligencias Médicas"2. En este tipo de pericias, el Servicio Médico Legal no se pronuncia sobre la existencia o no de una negligencia, dado que dicha calificación (como cuasidelito médico) le corresponde al sistema de justicia, sino que realiza una pericia que determina si hubo o no apego a Lex artis médica ${ }^{4}$, entendiendo por esta, según la definición de la Abogada Bárbara Quintana, al "conjunto de deberes que el facultativo debe respetar al realizar un acto médico, que están dados por la propia ciencia médica y cuya transgresión genera responsabilidad para éste, dando origen a los llamados juicios por mal praxis".

En sus orígenes, el quehacer del Departamento de Clínica Forense se centraba en la realizar principalmente Constataciones de Lesiones, pero desde finales de la década de los ochenta, se observó un incremento sostenido en la solicitud de informes 
de responsabilidad profesional, razón por la que en 1994 el SML oficializa la creación de una unidad especial destinada a resolver casos de mal praxis, denominada Unidad de Responsabilidad Sanitaria Médica, Odontológica y de los Servicios de Salud, más conocida como Unidad de Responsabilidad Médica (URM), la cual analiza casos relativos a todas las profesiones del área de la salud 5 .

La URM es una unidad centralizada, radicada en Santiago, que analiza casos provenientes de todo el país. Se debe señalar que el SML no posee la exclusividad en la realización de estos informes, dado que queda a arbitrio del juez o fiscal de la causa el lugar al que solicita el peritaje, pues existen otros centros referenciales, públicos y particulares, como son el Departamento de Medicina Legal de la Universidad de Chile, los peritos forenses del Listado de las Cortes de Apelaciones, algunas Sociedades Científicas y los centros de peritación médica particulares. Aún así, el SML el estamento que realiza la mayor parte de los peritajes de mal praxis en nuestro país.

El procedimiento a seguir cuando ingresa una solicitud de peritaje a la URM es el siguiente: luego de asignársele un número de pericia, el Encargado Técnico de la Unidad realiza un breve análisis del caso a fin de asignarlo a uno de los tres equipos de peritos existentes (médico, quirúrgico y obstétrico), donde a su vez se endosa a uno de sus peritos, médico o dentista según corresponda, el cual tendrá la responsabilidad de completar el estudio del caso, presentar su informe y conclusiones ante el resto del equipo, a fin de realizar un análisis y discusión grupal, obteniéndose entonces un informe pericial final, el cual se despacha a la entidad que lo solicitó ${ }^{6}$.

En la actualidad, se asume que la mayor parte de las demandas en estos casos dicen relación con el actuar de médicos. Sin embargo, al no disponer de información oficial sobre lo que está ocurriendo con otras profesiones del área de la salud en nuestro país, en particular de los odontólogos, surge la necesidad de revisar las bases de datos existentes, para así, indirectamente, caracterizar los casos de responsabilidad profesional que afectan a profesionales Odontólogos.

\section{Material y Método}

El presente trabajo es del tipo descriptivo, retrospectivo y analítico simple.
Los datos analizados fueron solicitados vía correo electrónico a la Unidad de Estadísticas del Servicio Médico Legal, sede Santiago, durante el primer semestre del año en curso ${ }^{7}$. Desde allí fueron remitidas dos bases de datos en formato Excel, la primera incluyendo número anual de ingresos entre 1994 y 2006 y la segunda, con el número de pericias efectuadas entre los años 2007 al 2012, individualizadas por número de pericia $\mathrm{y}$ año de ingreso, antecedentes generales del demandando, especialidad comprometida, datos sociodemográficos del demandante y estado de tramitación de la pericia.

Con la primera base de datos, únicamente se contabilizó el número total de ingresos, mientras que con la segunda, se procedió a la caracterización estadística de las solicitudes periciales ingresadas a la URM en el período 2007-2012 en términos de tipo de profesional afectado, especialidad de la pericia ingresada, patología de origen de la demanda, tipo de centro asistencial donde se origina la demanda, características de los pacientes demandantes y resultados del informe pericial generado.

\section{Resultados}

Entre 1994 y 2012, se registra el ingreso a la URM de un total de 7.000 casos, de los cuales 3.990 corresponden al período 2007-2012. La distribución anual de ingresos se encuentra representada en la Figura 1.

Hasta el año 2009, todas las solicitudes de informes periciales eran ingresadas a la base de datos de la URM. Posterior a esa fecha, sólo son ingresados a la base de datos aquellos casos que cuenten con todos los antecedentes requeridos para su realización, hecho que explica la disminución de ingresos observados entre el año 2009 y 2010. Cabe precisar que esta modificación no tuvo injerencia en las variables analizadas en el presente estudio.

Al clasificar las pericias ingresadas según profesional sanitario involucrado, se evidencia que los odontólogos ocupan el segundo lugar de las profesiones demandadas, muy por debajo de los médicos. Sólo $5 \%$ del total de casos ingresados involucran odontólogos, con un total de 203 casos en el período 2007-2012, distribuidos de la siguiente manera: 54 casos el 2007, 64 el 2008, 23 el 2009, 14 el 2010, 27 el 2011 y 21 el 2012. Para presentar las 


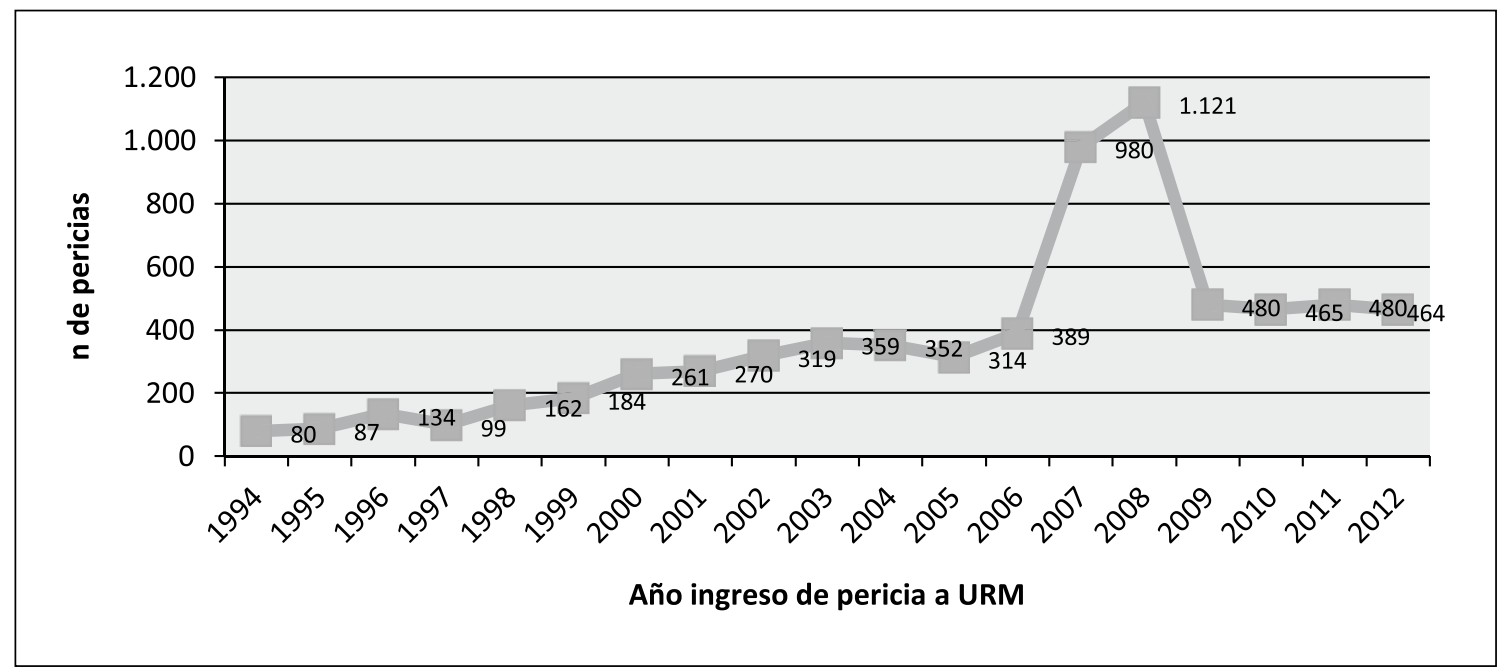

Figura 1. Solicitudes de pericias ingresadas a la Unidad de Responsabilidad Médica (URM) del Servicio Médico Legal (SML), período 1994-2012.

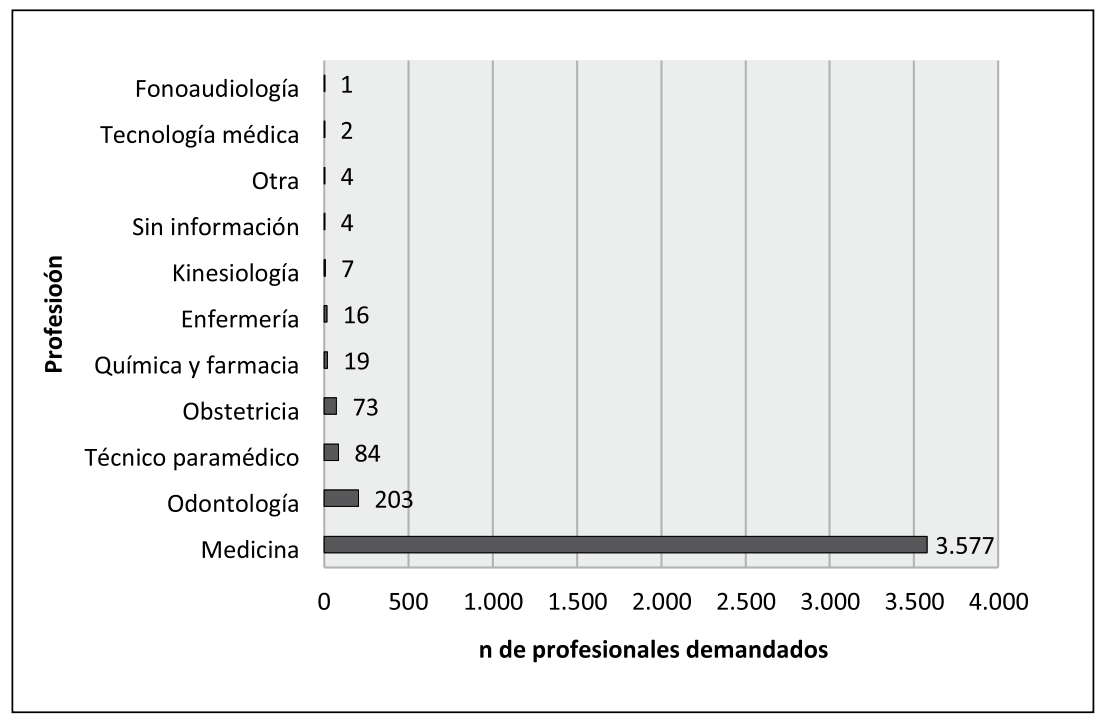

Figura 2. Solicitudes de pericias ingresadas a la URM del SML, según profesional sanitario demandado, período 2007-2012.

diferencias de ingreso de pericias según profesión del demandado, se presenta la Figura 2.

Ahora bien, si se realiza el análisis según especialidad comprometida, la ginecología y obstetricia es el área que más pericias genera (25\%), seguida por medicina interna $(13 \%)$, pediatría $(9 \%)$ y traumatología (7\%). Las pericias odontológicas son las que vienen a continuación, representando $5 \%$ del total de pericias ingresadas, tal como se observa en la Figura 3.
Al considerar el tipo de establecimiento donde se generaron los actos investigados, $72 \%$ del total de casos ingresados se genera en establecimientos públicos de salud, incluyéndose en esa categoría los hospitales universitarios y castrenses. Sin embargo, en el área odontológica este porcentaje es inverso, encontrándose que $69 \%$ de los casos se genera en establecimientos de salud privados.

Por otra parte, del total de pericias ingresadas entre los años 2007 y 2012, sólo 48,7\% se encon- 


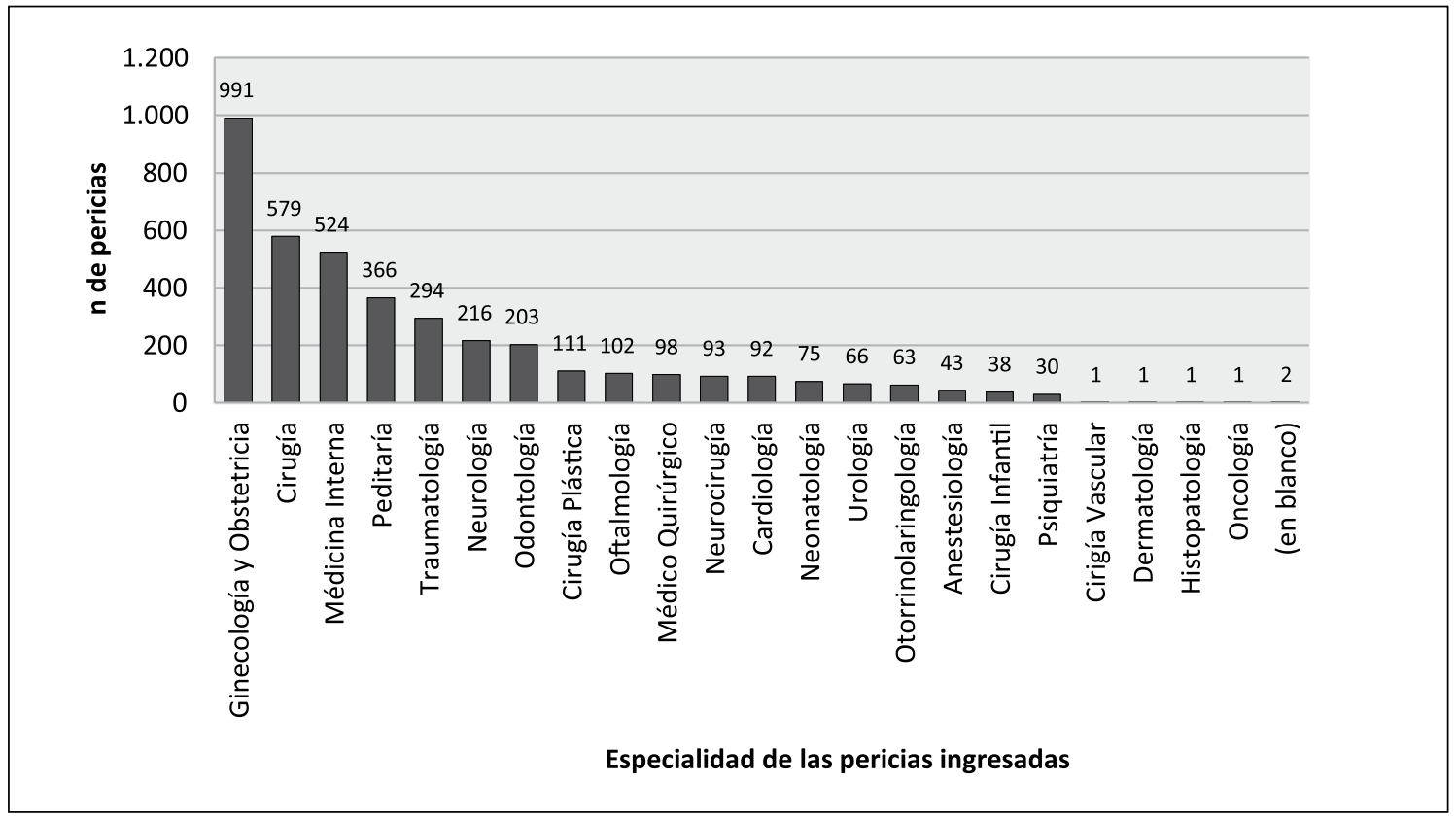

Figura 3. Distribución de las pericias ingresadas a la URM del SML, según especialidad involucrada, período $2007-2012$.

traban finalizadas a diciembre de 2013 (fecha de cierre de las estadísticas analizadas), y de ellas, $81 \%$ fue informada sin infracción a la Lex artis, 10\% como no concluyente y sólo $9 \%$ fue informada con infracción a la Lex artis. En contraposición, del total de pericias odontológicas ingresadas en el mismo período, $58 \%$ se encontraban finalizadas y de éstas, 34\% (40 casos) fueron informadas con infracción a la Lex artis.

$\mathrm{Al}$ ingreso de las solicitudes a la URM, además de clasificarse según área de especialidad comprometida, paralelamente se registra la causa de la demanda. En el área odontológica, al agrupar los diagnósticos asociados a las causas ingresadas, se observa que el origen de la demanda está mayoritariamente relacionado con razones quirúrgicas $(24 \%)$ e infecciosas $(21 \%)$, tal como se presenta en la Figura 4.

Respecto a las características de los pacientes afectados y demandantes, los datos analizados indican que en su mayoría son mujeres, con $82 \%$ versus $18 \%$ de demandantes varones. El $80 \%$ de los demandantes se concentra en edades intermedias de la vida, entre los 16 y 59 años, existiendo escasos casos en los rangos etarios más extremos.

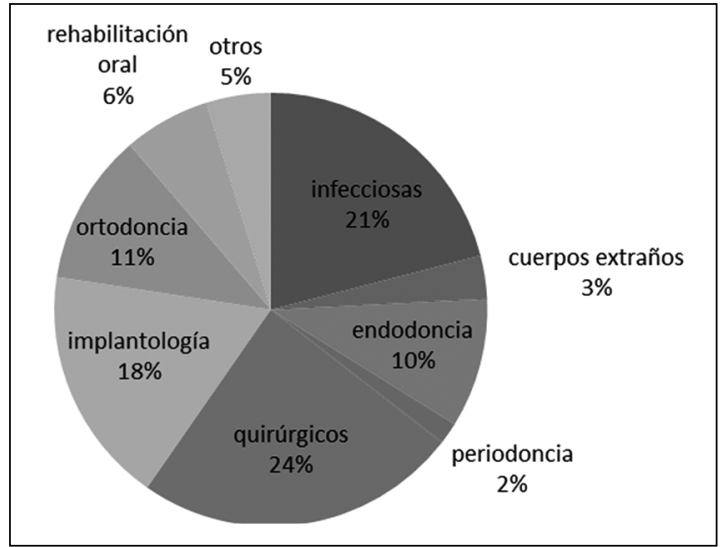

Figura 4. Distribución porcentual de las pericias odontológicas agrupadas por tipo de diagnóstico, período 2007-2012.

\section{Discusión}

En la última década se ha hablado cada vez más frecuentemente de la judicialización de la medicina, entendiendo por medicina los actos sanitarios efectuados por los distintos profesionales de la salud y no sólo por los profesionales médicos. Por otra parte, tradicionalmente ha sido el Servicio 
Médico Legal quien evalúa las pruebas periciales destinadas a determinar si se ha cumplido o no con la Lex artis, siendo esta institución la que realiza la mayor parte de los peritajes en esta área. Desafortunadamente, no se cuenta en la actualidad con datos estadísticos que permitan cuantificar esta afirmación, dejando abierta una línea de investigación complementaria al presente trabajo.

Ahora bien, las estadísticas del SML muestran efectivamente un aumento en el número de solicitudes de peritajes de responsabilidad sanitaria $y$, por ende, de los juicios a profesionales del área de la salud asociados a ellas. Y, si bien hasta el año 2008 se observa un alza de tipo exponencial, a partir de dicho año se visualiza una estabilización en el número de solicitudes, manteniéndose a la fecha en cifras que no superan los 500 casos por año, situación que puede ser explicada por diversas razones, como el cambio metodológico que efectuó la URM el 2009, relativo a los requisitos de ingreso de casos nuevos a la unidad, y que significó una brusca disminución de pericias ingresadas entre los años 2009-2010, la solicitud de pericias a entidades distintas al SML, la saturación del mercado, la insatisfacción de los demandantes y/o sus abogados con los resultados obtenidos.

El porcentaje mayoritario de las causas periciadas en la URM involucra a personal médico. Después de los médicos, los odontólogos son la segunda profesión que más juicios en causas de responsabilidad sanitaria poseen y la quinta área de especialidad más demandada, sin presentar un patrón de crecimiento constante. No se observan solicitudes de pericias para la especialidad odontológica cuyo demandado no sea odontólogo.

Considerando que según el Registro Nacional de Prestadores Individuales de la Superintendencia de Salud existen 15.334 odontólogos registrados en nuestro país a enero de $2014^{8}$, podría extrapolarse que menos de $1 \%$ de ellos se han visto involucrado en este tipo de pericias médico legales como demandado; sin embargo, al no contar con estadísticas sanitarias formales sobre causas ingresadas a tribunales por casos de mal praxis, existe la posibilidad de estar subestimando el número de odontólogos involucrados en dichos casos, y que por tanto, el porcentaje de dentistas involucrados sea mayor.

Por otra parte, los resultados observados indican que los casos periciados son mayoritariamente generados en establecimientos públicos, situación concordante con que más de $75 \%$ de la población del país es beneficiaria de FONASA ${ }^{9}$. Sin embargo, en el área odontológica esta situación es inversa, observándose que 69\% de las pericias se generaron en establecimientos privados. Esta situación se condice con un mayoritario desempeño de los odontólogos en el área privada y el escaso desarrollo de la odontología en el área pública ${ }^{10,11}$.

Asimismo en las pericias odontológicas, respecto al sexo del demandante, podemos señalar que el amplio predominio de las mujeres sobre los hombres está de acuerdo con la tasa de uso de los servicios sanitarios odontológicos, los cuales son mayoritariamente utilizados por mujeres ${ }^{12,13}$.

Respecto al resultado final de las pericias, llama poderosamente la atención que, contrariamente a la situación general, donde existe un claro predominio de resultados sin infracción a la Lex artis, en el área odontológica se determinó que en 35\% de los casos sí existía infracción a la Lex artis. Si bien este valor no puede considerarse como un sinónimo del porcentaje de casos que finaliza con un resultado adverso para el profesional demandado en Tribunales, lo cierto es que a la fecha, el SML constituye el gold standard en la realización de estos informes, por lo que en la mayor parte de los casos, una pericia con resultado negativo habitualmente se traduce en un resultado negativo para el profesional al finalizar el juicio.

La explicación de este 35\% debiese ser mutifactorial, pero si consideramos el hecho de que numerosas publicaciones en el área ${ }^{4,14,15,16}$ señalan que la principal causa de interposición de estas demandas es la percepción subjetiva de daño por parte del paciente, en el contexto de una relación profesional-paciente debilitada o inadecuada, esta cifra podría indicar que este tema es aún más crítico en el área odontológica, situación que pudiese ser un reflejo de la necesidad de fortalecer la bioética y deontología dentro de la enseñanza de la odontología $^{17,18,19,20}$, pues tal como señalan Zaror y Vergara "... (el método de enseñanza) ha llevado a una escasa motivación por la ética en la educación dental y a un escaso conocimiento de estos temas por parte del futuro profesional". Si se considera además el tema de las causas de las demandas odontológicas, podríamos estar presenciando evidencia del inadecuado manejo de pacientes con patologías quirúrgicas e infecciosas, afirmación sustentada en la escasa "cultura de derivación" que poseen los dentistas para efectuar referencias e interconsultas 
de sus pacientes, como asimismo, a un deficiente manejo de la farmacoterapia por parte de estos profesionales $^{21,22,23}$ Otras explicaciones podrían corresponder a que los peritos odontólogos sean más rigurosos que los peritos médicos en la determinación del apego a la Lex artis en sus informes o que, al predominar en los casos odontológicos la participación de un único profesional y a que habitualmente en odontoestomatología, las lesiones y complicaciones se manifiestan inmediatamente ${ }^{14}$, sea más sencillo establecer responsabilidades y relaciones de causalidad lineales entre el acto médico y el daño objetivado en el paciente. Finalmente, otra razón plausible es que, al igual que en la mayor parte de las carreras de la salud, los odontólogos cuentan con escasa formación de pregrado ${ }^{24}$ en temas médico legales ${ }^{25}$, lo que les genera una clara resistencia y negativo manejo al momento de enfrentar la judicialización de la relación con sus pacientes.

Por tanto, si bien el presente trabajo corresponde a una línea de investigación inicial en el tema de la mal praxis odontológica, sus resultados nos permiten presentar las siguientes recomendaciones, aplicables tanto para dentistas como para el personal sanitario en general: primeramente fortalecer la relación profesional-paciente, desarrollando modelos de alianza, en los que se ponga a disposición del paciente el tiempo y la información requerida para resolver dudas y conocer las eventuales complicaciones a las que se puede exponer con las respectivas acciones terapéuticas; segundo, y en necesaria conexión con lo anterior, tomar conciencia de la relevancia del tema desde ya, a fin de evitar que la judicialización de la odontología llegue a cifras homologables a las de los médicos; tercero, validar y certificar la obtención de competencias teóricas y técnicas que deban poseer los odontólogos al momento de egresar de sus carreras, al igual que los profesionales que ya se encuentran ejerciendo en el mundo profesional, $y$ finalmente, desarrollar docencia sobre responsabilidad profesional en el pregrado, pues al final del día, la medida más efectiva para evitar un juicio de mala praxis siempre ha sido la educación ${ }^{4,15,24}$.

\section{Referencias}

1. Ley Orgánica No 20.065 de modernización, regulación orgánica y planta del personal del Servicio Médico Legal del 21 de octubre de 2005. Disponible en www.bcn.cl
[Consultado el 04 de septiembre de 2013].

2. Szanthó G. Definiciones respecto al error en medicina. Rev Med Chile 2001; 129 (12): 1466-9. Disponible en www.scielo.cl [Consultado el 29 de agosto de 2013].

3. Pizarro C. Responsabilidad profesional médica: Diagnóstico y perspectivas. Rev Med Chile 2008; 136 (4): 539-43. Disponible en www.derechoysalud.udp.cl/ [Consultado el 21 de agosto de 2014].

4. Quintana B, Muñoz C. Responsabilidad médica: doctrina, legislación y jurisprudencia. Ediciones La Ley, Chile, 2004. ISBN: 956-7844-81-X.

5. Resolución Exenta $N^{\circ} 169$ del Servicio Médico Legal, de fecha 10 de marzo de 1994.

6. Presentación del Dr. Armando Valdés Herrera, ex Encargado Técnico de la URM del SML, en el VII Congreso de la Sociedad Iberoamericana de Derecho Médico, efectuado en la Universidad Diego Portales, septiembre de 2011.

7. Solicitud por Ley de Transparencia a la Superintendencia de Salud, Ingreso No AO006W-3006628, de fecha 04 de marzo de 2014.

8. Consulta sobre odontólogos en ejercicio del país, realizada en la página web de la Superintendencia de Salud www.supersalud.gob.cl/ [Consultado el 14 de enero de 2014].

9. Boletín estadístico 2010-2012 de FONASA, disponible en www.fonasa.cl/portal_fonasa/site/artic/20140607/ pags/20140607230746.html [Consultado el 01 de diciembre de 2014].

10. Informe Catastro Nacional de Recursos Humanos Odontológicos en el SNSS. División de Rectoría y Regulación Sanitaria, Departamento de Salud Bucal, Ministerio de Salud, 2003.

11. Cabello R, Rodríguez G, Tapia R, Jara G, Soto L, Venegas C. Recursos humanos odontológicos y necesidades de tratamiento de caries en adolescentes de 12 años en Chile. Rev Clin Periodoncia Implantol Rehabil Oral 2011; 4 (2): 45-9.

12. Bertakis KD, Azari R, Helms LJ, Callahan EJ, Robbins JA. Gender differences in the utilization of health care services. J Fam Pract 2000; 49 (2): 147-52.

13. Edelstein BL. Disparities in oral health and access to care: findings of national surveys. Ambul Pediatr 2002; 2 (2 Suppl): 141-7.

14. Perea B, Labrajo ME, Santiago A, Albarrán ME. Responsabilidad profesional en odontología. Rev Esp Med Legal 2013; 39 (4): 149-56.

15. Garay O. Tratado de responsabilidad civil en las especialidades médicas. Editorial Errepar, Argentina, 2009. ISBN: 9870109349.

16. Enríquez J. El contrato de asistencia médica y la prue- 
ba de la culpa médica, disponible en www.academia. edu/7398946/EL_CONTRTO_DE_ASISTENCIA_ MEDICA_Y_LA_PRUEBA_DE_LA_CULPA_MEDICA [Consultado el 24 de agosto de 2014].

17. Izzeddin R, Spina M, Tosta E. Dimensión ética en la práctica odontológica: visión personalista. Acta Bioethica 2010; 16 (2): 207-10.

18. Torres-Quintana MA, Romo F. Bioética y ejercicio profesional de la odontología. Acta Bioethica 2006; 12 (1): 65-74.

19. Triana J. La ética: un problema para el odontólogo. Acta Bioethica 2006; 12 (1): 75-80.

20. Zaror C, Vergara C. Ética en el currículo de las carreras de odontología. Acta Bioethica 2008; 14 (2): 212-8.

21. Narváez CG, Vicentela LA. Actualidad y perspectiva del currículum de odontología. Rev Cub Ed Médica Superior 2011; 25 (4): 515-24.
22. Der C, Salin MP, Iñiguez M, Valenzuela P. Realidad de la derivación a otorrinolaringología desde la atención primaria. Rev Otorrinolaringol Cir Cabeza Cuello 2006; 66 (2): 95-102. Disponible en www.scielo.cl [Consultado el 12 de agosto de 2014].

23. Berge TI. General practitioners and dental students' decisions on third-molar diagnoses, treatment, and referrals. Acta Odontol Scand 1993; 51 (3): 171-81.

24. González L, Inzunza J, Bustos L, Vallejos C, Gutiérrez R. Docencia e Investigación en medicina legal: situación actual y desafíos para las Facultades de Medicina de Chile. Rev Med Chile 2005; 133: 805-12.

25. Carrasco P. Responsabilidad penal profesional (tesis), 2005, disponible en biblioteca.uandes.cl/documentos/ DesplegarTesis.asp?nombre=C:/www/biblioteca/documentos/Pcarrasco.xml [Consultado el 05 de septiembre de 2014]. 